\title{
Validity Of The Self-Reported Domains Of Subjective Extent Of Nonadherence (DOSE-Nonadherence) Scale In Comparison With Electronically Monitored Adherence To Cardiovascular Medications
}

This article was published in the following Dove Press journal:

Patient Preference and Adherence

\author{
Talea Cornelius (iD ${ }^{\prime}$ \\ Corrine I Voils $\mathbb{D}^{2,3}$ \\ Redeana C Umland' \\ Ian M Kronish (iD) \\ 'Center for Behavioral Cardiovascular \\ Health, Department of Medicine, \\ Columbia University Irving Medical \\ Center, New York, NY 10032, USA; \\ ${ }^{2}$ William S Middleton Veterans Memorial \\ Hospital, Madison, WI 53705, USA; \\ ${ }^{3}$ Department of Surgery, University of \\ Wisconsin-Madison School of Medicine \\ and Public Health, Madison, WI \\ 53792, USA
}

Background: Self-report measures are important tools for assessing adherence to medication. Many of these scales, however, combine the extent of and reasons for nonadherence in one instrument, and have limited reliability and validity. The present study was the first to assess the convergent validity of the self-reported Domains of Subjective Extent of Nonadherence (DOSE-Nonadherence) scale with electronically measured adherence to a single cardiovascular medication.

Methods: English- and Spanish-speaking patients evaluated for acute coronary syndrome $(\mathrm{N}=165 ; n=68$ and $\mathrm{n}=97$, respectively) were recruited from an urban academic emergency department. Post-hospital discharge, participants were mailed a medication bottle with an electronic cap (eCAP) that recorded bottle openings. At 1 month, participants completed the 3-item DOSE-Nonadherence scale, which assessed the extent to which patients missed, skipped, or did not take the eCAP-monitored medication over the past 7 days. Correlations, sensitivity, and specificity were calculated using eCAP-monitored correct dosing adherence over the same 7-day time period as the reference standard.

Results: The most commonly assessed medication was aspirin (70.91\%). Correlations between self-reported and electronically monitored adherence were low-to-moderate: English-speaking participants $(\mathrm{n}=68), r=0.24, \quad p=0.046$; Spanish-speaking participants $(\mathrm{n}=97), r=0.18, p=0.071$. Sensitivity was low (0.47 English, 0.28 Spanish) and specificity was moderate (0.77 English, 0.88 Spanish).

Conclusions: The DOSE-Nonadherence scale was associated with electronically monitored adherence to a single daily cardiovascular medication in English-speaking participants, but had weak diagnostic properties when using electronic adherence as the reference standard.

Keywords: medication adherence, self-report, nonadherence, validity, electronic monitoring

\section{Introduction}

Medication nonadherence represents a significant challenge to the management of chronic disease. It is both highly prevalent and associated with a wide range of negative outcomes, including increased healthcare spending, hospitalizations, and mortality. ${ }^{1-11}$ Tools are needed to measure medication nonadherence in research and clinical settings. Indeed, a recent systematic review concluded that first measuring nonadherence and then targeting adherence interventions at nonadherent patients is a key component of successful interventions to improve medication adherence. ${ }^{12}$
Correspondence: Talea Cornelius Center for Behavioral Cardiovascular Health, Columbia University Irving Medical Center, 622 West 168th Street PH9-319, New York, NY 10032, USA $\mathrm{Tel}+1212-304-5215$

Email tmc2184@cumc.columbia.edu 
Self-report measures are an attractive option for measuring nonadherence and identifying nonadherent patients, given low cost and feasibility of administering these assessments. It is also possible that patients who are willing to endorse nonadherence may be more amenable to interventions. Yet, no "gold standard" self-report measure exists. ${ }^{13}$ Many of these measures have limited reliability and/or validity, ${ }^{14,15}$ in part because they measure both extent of nonadherence and reasons for nonadherence as a unidimensional construct, even though these are separate (but related) considerations. ${ }^{14}$

To address the limitations of many self-reported adherence measures, Voils et al ${ }^{14,16}$ developed a 2-domain measure comprising 3 items to assess the extent of nonadherence to medications, along with a separate, disease-specific method of capturing reasons for nonadherence, the Domains of Subjective Extent of Nonadherence (DOSE-Nonadherence) scale. In initial work in patients prescribed antihypertensive medications, the extent of nonadherence measure demonstrated good internal consistency reliability $(\alpha=0.84)^{14}$ and remained reliable over a 12-month $\operatorname{span}(\alpha \mathrm{S}=0.75-0.90) .{ }^{17}$ The extent of nonadherence was also associated with higher blood pressure $(r \mathrm{~s}=0.27, p \mathrm{~s}<0.001) .{ }^{14}$ Additionally, the extent of nonadherence measure demonstrated a strong correlation with the widely used Morisky Medication Adherence Scale (8item MMAS), ${ }^{18}$ another self-report measure of nonadherence. In the study that compared the DOSE-Nonadherence scale and MMAS, only the DOSE-Nonadherence scale was associated with blood pressure. ${ }^{14}$ More recently, the DOSE-Nonadherence scale showed high reliability across repeated assessments in a sample of patients taking oral medications for hepatitis C. ${ }^{19}$ Furthermore, endorsement of any nonadherence to medications prescribed for dyslipidemia was shown to be related to higher serum total and LDL cholesterol levels, ${ }^{17,20}$ and changes in self-reported extent of nonadherence exhibited predictive validity for changes in serum total cholesterol over time. ${ }^{17}$ These results are promising for both reliability and validity of the scale.

Thus far, the DOSE-Nonadherence scale has not been compared to other approaches to measuring nonadherence to daily medications (i.e. missed doses). The present study is intended to assess the convergent validity of the DOSE-Nonadherence scale by comparing self-reported nonadherence, in terms of daily missed or skipped doses, to electronically monitored daily adherence to cardiovascular medications in a sample of patients recently evaluated for a suspected acute coronary syndrome (ACS). Electronic monitoring is widely regarded as a gold standard measure of adherence to daily medications because it is more objective than self-report (which may be impacted by, e.g. recall bias or social desirability bias) and because it has shown little-to-no reactivity (i.e. does not impact medication-taking behaviors). ${ }^{2,7,21}$ Electronic monitoring may also be ideally suited to evaluating the concurrent validity of the DOSE-Nonadherence scale because, even though the extent of medication nonadherence scale measures ingestion of daily medications and electronic monitoring measures daily bottle openings, both measurement approaches evaluate day-to-day medicationtaking behavior (commonly known as regimen implementation). ${ }^{22}$

\section{Methods}

\section{Design}

This paper presents secondary analyses from an observational cohort study (REactions to Acute Care and Hospitalization; REACH) that examines the development and impact of cardiac-induced posttraumatic stress disorder among patients presenting to the emergency department (ED) with a suspected ACS. ${ }^{23}$ For the present study, we report cross-sectional analyses comparing self-reported and electronically monitored adherence to cardiovascular medication for a 7-day period that occurred approximately 30 days post-discharge.

\section{Participants}

Inclusion criteria for REACH included presentation to the ED of a tertiary care hospital (Columbia-New York Presbyterian Hospital, New York, NY) with a suspected ACS (unstable angina or non-ST elevation myocardial infarction by the treating ED physicians). Exclusion criteria were non-English- or Spanish-speaking and any cognitive or psychiatric impairment that would impede study participation (for further details on REACH design, see Birk et $\left.\mathrm{al}^{23}\right)$. Participants $(\mathrm{N}=165)$ were included in the present study if they completed the self-reported nonadherence scale and if they completed electronic medication adherence monitoring substudy (i.e. participants had to be prescribed aspirin or another cardiovascular medication ${ }^{23}$ ) in the first month after the index hospitalization (the period corresponding with the self-report adherence assessment). 


\section{Procedures}

Potentially eligible participants were identified via treating emergency medicine physicians and approached by a research assistant in the ED to confirm eligibility, gauge interest, and obtain informed consent. A brief assessment took place in-ED (e.g. demographics) and again a median of 3 days post-ED-discharge (either in-person while inpatient or via telephone at home). At the post-ED follow-up visit, participants prescribed at least one cardiovascular medication were invited to utilize an electronic pill cap (eCAP, Information Mediary Corp., Ottawa, Canada) to monitor their medication adherence. The eCAP, which looks similar to a conventional pill bottle cap and fits on a standard size medication bottle, records the date and time when the cap is opened. Those who agreed were mailed an eCAP-covered medication bottle after discharge from the hospital. They were instructed to place one of their cardiovascular medications, preferably aspirin, in the bottle, and to take the medication in the manner prescribed by their doctor. No other counseling about medication adherence was provided.

Participants completed a telephone interview again at a minimum of 1 month post-discharge. This assessment included the measure of self-reported medication nonadherence with a recall period of the past 7 days. During this phone call, participants were asked to return the eCAP in a stamped envelope that they would soon receive in the mail. Participants did not receive any advice about how to take their cardiovascular medication during this phone call. The study protocol conforms to the ethical guidelines of the 1975 Declaration of Helsinki, and all materials were approved by the Columbia University Irving Medical Center Institutional Review Board (IRB). All participants provided written informed consent before completing study procedures.

\section{Measures}

\section{Extent Of Nonadherence: Self-Report}

The extent of nonadherence to cardiovascular medication over the past 7 days was measured using the DOSENonadherence scale, which comprises three items scored from 1, "None of the time," to 5, "All of the time." Items included: "In the past 7 days, how often did you ... miss a dose of your heart medication?" "[...] skip a dose of your heart medications?" and "[...] not take your heart medication as prescribed by your doctor?"14,16,19 For those using an eCAP, research coordinators substituted "heart medications" with the name of the cardiovascular medication being tracked by the eCAP when administering the extent of nonadherence questionnaire. The DOSENonadherence scale was modified by the study in two ways. The third item added "as prescribed by your doctor" to the end of the question; this change occurred after multiple Spanish-speaking participants requested clarification of the third item during user testing in our study population. The response option "all of the time" was modified from "every time" for consistency with other response scales in the survey.

The questionnaire was translated into Spanish in an iterative process. First, measures were translated from English to Spanish by an IRB-certified translator. Next, translations were reviewed and user tested with a group of experienced bilingual research assistants representing a diverse range of dialects to maximize understanding in all Spanish-speaking populations (e.g. Spain, Dominican Republic, Cuba, Puerto Rico, Colombia, Ecuador). During this review, a final Spanish version was agreed upon through consensus.

The mean of the 3 items was computed to form a continuous assessment of the extent of nonadherence with a range of scores from 1 to 5 (for English-speaking participants, $\alpha=0.95$; for Spanish-speaking participants, $\alpha=0.87$ ). The score was also dichotomized to indicate 0 , no endorsement of nonadherence, and 1, any nonadherence (i.e. a response of 2 or greater on any of the 3 items).

\section{Extent Of Nonadherence: Correct Dosing Adherence Via Electronic Monitor}

Participants were asked to track one of their daily cardiovascular medications - preferably aspirin for ease of between-patient comparisons - using eCAPs. They were otherwise told to take the medication as prescribed by their doctor. The prescribed frequency of dosing was determined based on a review of the discharge medication list in the electronic medical record. Adherence was calculated as the percent of days a participant opened the eCAPs bottle the correct number of times. For example, if a medication was prescribed once a day and that individual opened the bottle once, then they were considered adherent on that day. In contrast, if a participant opened the bottle twice that day or not at all, then they were considered nonadherent on that day. Data were censored if the participant was in the hospital or had an explained gap (e.g. doctor advised participant to stop taking medication). 
Percent adherence for the 7 days prior to the self-report was computed. For example, if a participant opened their bottle the prescribed number of times, as determined by eCAPs, on 7 of 7 days, they were assigned a score of 1.00 (100\% taken as prescribed). If a participant opened their bottle the prescribed number of times on only 2 of 7 days, they were assigned a score of $0.29(28.57 \%$ taken as prescribed). Note that participants with any data were included (e.g. if eCAPs had been started only 5 days prior, such that 5 days of data were available, then 5 was the denominator for the percent adherence calculation). Continuous percent adherence scores were also dichotomized such that 0 indicated perfect adherence (i.e. 100\% taken as prescribed on the days they were monitored), whereas 1 indicated any nonadherence during the monitoring period (i.e. any percent adherence ranging from $0 \%$ to less than 100\%). For use in sensitivity analyses, we additionally computed adherence as opening the eCAP bottle at least the prescribed number of times (e.g. opening a bottle twice for a once-per-day medication was considered adherent). "At least" was chosen to correspond to the fact that the self-report measure assesses missed doses only, whereas correct dosing adherence penalizes both missed doses and extra doses.

\section{Data Analysis Strategy}

Extent of nonadherence (self-reported and as measured by eCAPs; continuous and dichotomous) was examined using descriptive statistics. For correlational analyses, self-reported nonadherence was reverse coded so that a 1 represented the lowest adherence and a 5 indicated the highest adherence. This was done to facilitate comparisons with the eCAPs measure of adherence. Associations between self-report and eCAPs measures were tested using Spearman correlations due to high levels of skewness in the data.

The sensitivity and specificity of the self-report measure for detecting nonadherence were calculated using electronically monitored adherence of less than $100 \%$ as the reference standard. A cutpoint of $100 \%$ was selected as it best corresponded to the self-report response options, where an answer of 1 on each item was suggestive of perfect adherence, but any endorsement of a response option 2-5 indicated at least some nonadherence. Sensitivity indicates the ability of the self-report measure to identify "true positives" (i.e. of those who were nonadherent on eCAPS, what proportion screened as nonadherent on the self-report measure?). Specificity indicates the ability of the self-report measure to identify "true negatives" (i.e. of those who were adherent on eCAPs, what proportion screened adherent on the self-report measure?). Positive and negative likelihood ratios (LRs) were also calculated. LRs indicate how a positive (nonadherence indicated) or negative (no nonadherence indicated) screen impacts the likelihood that a patient is nonadherent. Positive LRs (LR+) are calculated as sensitivity/(1-specificity), and negative LRs (LR-) as specificity/(1-sensitivity). A LR + of more than 10 is considered to have a large effect on posttest probability of being a "true positive" if a patient screens positive on self-reported nonadherence. ${ }^{24}$ A LR + of 5 to 10 indicates a moderate effect, and 2 to 5 a small effect ( 1 indicates no change). Similarly, a LR- of less than 0.1 is considered to have a large effect on posttest probability of being a "true negative" if a patient does not report nonadherence, 0.1 to 0.2 a moderate effect, and 0.2 to 0.5 a small effect. ${ }^{25}$ Analyses were conducted separately for English- and Spanish-speaking patients.

\section{Results}

Demographic characteristics for the $\mathrm{N}=165$ included in the present study can be seen in Table 1. Participants were a mean of 61.87 years old $(\mathrm{SD}=11.16), 54.55 \%$ were male, and $41.21 \%$ spoke English as a first language. The most commonly electronically monitored medication was aspirin $(70.91 \%)$, and the most common dose frequency was once per day ( $92.12 \%$; the rest were twice per day).

Mean self-reported nonadherence during the 7 days prior to the 1-month assessment was low $(\mathrm{M}=1.27$, $\mathrm{SD}=0.62$ ). In other words, participant tended to report that they "missed," "skipped," or "did not take medications as prescribed" none of the time (recall that $1=$ "none of the time"). Only 47 participants (28.48\%) reported any nonadherence. Mean continuous adherence as measured by eCAPS during the 7 days prior to the 1-month assessment (i.e. percent bottle openings, as prescribed) was $66.56 \%$ ( $\mathrm{SD}=36.29 \%$ ). In other words, the eCAPs bottle was opened the correct number of times on $66.56 \%$ of days measured. More than half of the participants $(61.82 \%)$ were considered nonadherent (i.e. incorrect number of openings on at least 1 day).

\section{Continuous Measures Of Adherence}

The continuous measure of self-reported nonadherence to cardiovascular medications was correlated with the eCAPmeasured adherence. Recall that self-report was reverse coded for these correlations such that a positive correlation indicates convergence between the measures. For the 68 
Table I Characteristics Of Study Participants

\begin{tabular}{|c|c|c|c|c|}
\hline & & \multicolumn{3}{|l|}{ M (SD) or \% } \\
\hline & & Overall $(N=165)$ & English Speaking $(n=68)$ & Spanish Speaking $(n=97)$ \\
\hline Age in years & & $61.87(11.16)$ & $60.10(11.43)$ & $63.10(10.78)+$ \\
\hline \multirow[t]{2}{*}{ Sex } & Male & $54.55 \%$ & $57.35 \%$ & $47.42 \%$ \\
\hline & Female & $45.45 \%$ & $42.65 \%$ & $52.58 \%$ \\
\hline \multirow[t]{3}{*}{ Race } & Black & $18.79 \%$ & $42.65 \%$ & $2.06 \% * *$ \\
\hline & White & $8.48 \%$ & $16.18 \%$ & $3.09 \%$ \\
\hline & Other & $72.73 \%$ & $41.17 \%$ & $94.85 \%$ \\
\hline \multirow[t]{2}{*}{ Ethnicity } & Hispanic & $66.67 \%$ & $29.41 \%$ & $92.78 \%$ \\
\hline & Non-Hispanic & $34.33 \%$ & $70.59 \%$ & $7.22 \%$ \\
\hline \multirow[t]{4}{*}{ Education } & High school or Less & $60.00 \%$ & $39.71 \%$ & $74.23 \% * *$ \\
\hline & Trade school/some college & $16.97 \%$ & $26.47 \%$ & $10.31 \%$ \\
\hline & College degree & $12.73 \%$ & $11.76 \%$ & $13.40 \%$ \\
\hline & Graduate degree & $10.30 \%$ & $22.06 \%$ & $2.06 \%$ \\
\hline \multirow[t]{2}{*}{ English as a first language } & No & $58.79 \%$ & & \\
\hline & Yes & $41.21 \%$ & & \\
\hline \multirow[t]{2}{*}{ Confirmed ACS } & Yes & $33.33 \%$ & $35.29 \%$ & $31.96 \%$ \\
\hline & No & $66.67 \%$ & $64.71 \%$ & $68.04 \%$ \\
\hline
\end{tabular}

Notes: $+P<0.10, * * P<0.01$.

English-speaking participants, this correlation was, $r=0.24$, $p=0.046$, and for the 97 Spanish-speaking participants it was, $r=0.18, p=0.071$.

\section{Sensitivity Analysis}

When substituting the correct dosing adherence measure with an electronic measure in which opening the bottle "at least" the prescribed number of times was adherent (i.e. not counting days on which participants took extra doses as nonadherent days), the correlation of self-report with eCAPs was slightly lower for English-speaking participants, $r=0.22, p=0.067$, but slightly higher for Spanishspeaking participants, $r=0.24, p=0.016$.

\section{Dichotomous Measure Of Adherence}

Proportion of participants detected as $100 \%$ adherent by eCAPs stratified by self-reported adherence can be seen in Figure 1. Details regarding sensitivity, specificity, and positive and negative LRs can be seen in Table 2. Participants who self-reported at least some nonadherence were more likely to be nonadherent by electronic monitoring than those who selfreported being adherent. Sensitivity was lower than specificity in both English-speaking participants ( 0.47 versus 0.77 ) and Spanish-speaking participants $(0.28$ versus 0.88$)$.

\section{Sensitivity Analysis}

When substituting the correct dosing adherence measure with an electronic measure in which opening the bottle "at least" the prescribed number of times was adherent, sensitivity and specificity were 0.48 versus 0.74 for Englishspeaking participants, respectively, and 0.33 versus 0.89 for Spanish-speaking participants.

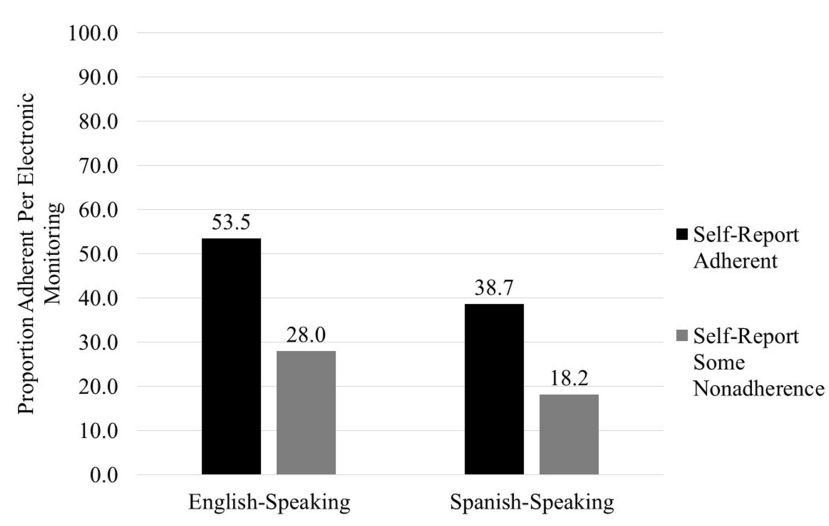

Figure I Comparison of adherence classification by the 3-item extent of nonadherence questionnaire with electronically monitored adherence, stratified by participant language.

Note: Medications were electronically monitored using eCAPs (English speaking, $\mathrm{n}=68$; Spanish speaking, $\mathrm{n}=97$ ). 
Table 2 Sensitivity, Specificity, And Likelihood Ratios (LRs) Of The Extent Of Nonadherence Questionnaire For Detecting Medication Nonadherence

\begin{tabular}{|l|l|l|l|l|}
\hline & Sensitivity & Specificity & LR+ & LR- \\
\cline { 2 - 5 } & Est $[95 \% \mathbf{C I}]$ & Est [95\% Cl] & Est [95\% CI] & Est [95\% CI] \\
\hline Overall $(\mathrm{N}=165)$ & $0.35[0.26,0.45]$ & $0.83[0.71,0.91]$ & $2.02[0.80,3.24]$ & $0.78[0.64,0.93]$ \\
English-speaking $(n=68)$ & $0.47[0.31,0.64]$ & $0.77[0.58,0.90]$ & $2.03[0.52,3.54]$ & $0.69[0.43,0.94]$ \\
Spanish-speaking $(n=97)$ & $0.28[0.18,0.41]$ & $0.88[0.72,0.97]$ & $2.32[0.00,4.67]$ & $0.82[0.65,0.98]$ \\
\hline
\end{tabular}

Notes: Any (vs no) self-reported nonadherence over the past 7 days on the extent of nonadherence questionnaire indicated a positive screen. $<100 \%$ (vs $100 \%$ ) adherence over the past 7 days, as electronically monitored by eCAPs, indicated a true positive for nonadherence.

\section{Discussion}

The present study provided modest support for the convergent validity of the DOSE-Nonadherence scale with electronically monitored adherence to daily cardiovascular medications as the reference standard in a cohort of patients with suspected ACS. The self-report measure had low sensitivity and moderate specificity for identifying nonadherent patients per electronic monitoring.

The DOSE-Nonadherence scale was developed to address the limited reliability and/or validity of self-report measures that often conflate reasons for nonadherence with the behavior of nonadherence. ${ }^{14,15}$ A recent systematic review of these previously developed self-report adherence questionnaires (14 standardized questionnaires, 11 unnamed) found comparable correlations between selfreported and electronically monitored medication adherence in diverse patient populations. ${ }^{26}$ Also similar to other self-report questionnaires, participants were more likely to report being adherent by self-report as compared to electronic-measurement; only $29 \%$ reported at least some nonadherence, whereas $62 \%$ were found to have at least some nonadherence by eCAPs. Considering the present study alongside prior research on this self-report scale, which provided evidence for predictive validity regarding health outcomes and response to treatment, ${ }^{17}$ the DOSENonadherence scale has demonstrated good statistical properties for research. The fact that this is a brief, 3item scale that is inexpensive and easy to implement with acceptable specificity may make it desirable to incorporate into research settings.

The sensitivity of the dichotomized DOSENonadherence scale was low (i.e. ability to detect "true positives" from the entire population of nonadherent patients). This is not necessarily surprising given a general tendency of patients to over-report adherence across selfreported medication adherence questionnaires. Furthermore, eCAPs assessed bottle openings, not actual ingestion of medications, and so correlations could be attenuated by the lack of conceptual overlap regarding the behavior being measured (i.e. self-reported ingestion vs the behavior of bottle openings). There may also be other factors contributing to the lack of correspondence, such as social desirability bias, inability to recall behavior (particularly if cognitive load is high), or any other number of cognitive processes.

Although the scale was poor at identifying all participants who were nonadherent by electronic monitoring, $72.0 \%$ of English-speaking participants and $81.8 \%$ of Spanish-speaking participants who endorsed any nonadherence were also nonadherent per the electronic monitor. Thus, this scale may be useful for identifying nonadherent patients in clinical settings. In addition, the fact that these patients disclosed nonadherence may make them more amenable to adherence interventions. Pairing the extent of nonadherence scale items with items to assess reasons for nonadherence, ${ }^{14,27}$ as the DOSE-Nonadherence scale was designed, ${ }^{27}$ may identify targets for adherence interventions.

There were a number of strengths of our analyses. We rigorously measured electronic adherence in a diverse patient population inclusive of those with low socioeconomic status. We additionally computed a measure of electronic adherence for use in sensitivity analyses that did not count patients as nonadherent if they took extra doses. There were also some limitations. eCAPs measure bottle openings, and it is possible that participants did not actually ingest the medication when bottles were opened. Alternatively, participants may have taken doses without using the eCAP. Results may not generalize to patients prescribed other types of medications, to regimens that comprise multiple cardiovascular medications, or to patients recruited outside of urban ED settings. Future studies should explore whether self-report scales are best used to measure adherence to a single medication or to regimen adherence more broadly. ${ }^{28}$ The DOSE-Nonadherence scale 
focuses on missed doses; extra doses or wrong timing of doses may be important as part of adherence measurement for other self-report adherence questionnaires. It is possible that 30-day adherence more accurately reflects long-term adherence, however, in cognitive interviews conducted for scale construction and item generation, participants felt that asking about adherence over the past 7 days led to more accurate recall and greater sensitivity to nonadherence. ${ }^{14}$ To measure long-term patterns, ideally, the DOSE-Nonadherence scale should be assessed repeatedly. The scale was developed using English speakers and the Spanish version may have been interpreted differently by participants. The DOSE-Nonadherence scale had lower reliability and validity in the Spanish version of the present study. Future research is needed to refine and further validate this scale in Spanish-speaking populations. The sample size for both samples was modest, which limited power for precise determination of diagnostic utility. The DOSE-Nonadherence scale was modified slightly from its original version in response to questions from study participants during user testing in our study population; rather than asking about "not taking" doses according to the original version, participants were asked about not taking doses "as prescribed by your doctor." Thus, extrapolation of the current findings to use of the original questionnaire should be done cautiously. However, although "as prescribed" could refer to numerous aspects of dose administration (e.g. time of day, food instructions, etc.), the cardiovascular medications assessed (such as aspirin) come with few dosing requirements. Furthermore, this wording is in parallel to our eCAPs measure of correct dosing adherence, and results did not change in sensitivity analyses when patients were not penalized for taking extra doses.

\section{Conclusion}

Our analyses demonstrate that the extent of nonadherence domain of the DOSE-Nonadherence scale has modest convergent validity with a behavioral measure of medication adherence, providing support for the usefulness of this tool as a measure of medication adherence in research settings. Future studies should assess the convergent validity of this measure versus behavioral measures of regimen implementation in other patient populations and should evaluate the usefulness of this tool in clinical settings in larger samples.

\section{Acknowledgments}

This work was supported by the National Heart, Lung, and Blood Institute (NHLBI; R01HL117832, and R01HL123368). The content is solely the responsibility of the authors and does not necessarily represent the official view of the National Institutes of Health. The funding body had no role in the design of the study, collection and interpretation of data, or in writing this manuscript. Effort on this manuscript was also made possible by a Research Career Scientist award from the Health Services Research and Development service of the Department of Veterans Affairs (VA; grant number RCS 14-443). The views expressed in this article are those of the authors and do not necessarily reflect the position or policy of the VA or the United States Government.

\section{Disclosure}

Dr Ian Kronish reports grants from NHLBI. Dr Corrine Voils reports grants from Health Services Research and Development Service of the Department of VA during the conduct of the study. The authors report no other conflicts of interest in this work.

\section{References}

1. Dunbar-Jacob J, Mortimer-Stephens M. Treatment adherence in chronic disease. J Clin Epidemiol. 2001;54(12):S57-S60. doi:10.10 16/s0895-4356(01)00457-7

2. Osterberg L, Blaschke T. Adherence to medication. New Engl J Med. 2005;353(5):487-497. doi:10.1056/NEJMra050100

3. DiMatteo MR. Variations in patients' adherence to medical recommendations: a quantitative review of 50 years of research. Med Care. 2004;42(3):200-209. doi:10.1097/01.mlr.0000114908.90348.f9

4. Gellad WF, Thorpe CT, Steiner JF, Voils CI. The myths of medication adherence. Pharmacoepidemiol Drug Saf. 2017;26(12):1437-1441. doi:10.1002/pds.4334

5. Tajeu GS, Kent ST, Kronish IM, et al. Trends in antihypertensive medication discontinuation and low adherence among medicare beneficiaries initiating treatment from 2007 to 2012. Hypertension. 2016;68(3):565575. PMC5215087. doi:10.1161/HYPERTENSIONAHA.116.07720

6. Chowdhury R, Khan H, Heydon E, et al. Adherence to cardiovascular therapy: a meta-analysis of prevalence and clinical consequences. Eur Heart J. 2013;34(38):2940-2948. doi:10.1093/eurheartj/eht295

7. Kronish IM, Ye S. Adherence to cardiovascular medications: lessons learned and future directions. Prog Cardiovasc Dis. 2013;55(6):590600. PMC3639439. doi:10.1016/j.pcad.2013.02.001

8. Simpson SH, Eurich DT, Majumdar SR, et al. A meta-analysis of the association between adherence to drug therapy and mortality. BMJ. 2006;333(7557):15. PMC1488752. doi:10.1136/bmj.38875.675486.55

9. DiMatteo MR, Giordani PJ, Lepper HS, Croghan TW. Patient adherence and medical treatment outcomes: a meta-analysis. Med Care. 2002;40(9):794-811. doi:10.1097/00005650-200209000-00009

10. Viswanathan M, Golin CE, Jones CD, et al. Interventions to improve adherence to self-administered medications for chronic diseases in the united states: a systematic review. Ann Intern Med. 2012;157 (11):785-795. doi:10.7326/0003-4819-157-11-201212040-00538 
11. Sokol MC, McGuigan KA, Verbrugge RR, Epstein RS. Impact of medication adherence on hospitalization risk and healthcare cost. Med Care. 2005;43(6):521-530. doi:10.1097/01.mlr.0000163641.86 870.af

12. Allemann SS, Nieuwlaat R, Navarro T, Haynes B, Hersberger KE, Arnet I. Congruence between patient characteristics and interventions may partly explain medication adherence intervention effectiveness: an analysis of 190 randomized controlled trials from a Cochrane systematic review. J Clin Epidemiol. 2017;91:70-79. doi:10.1016/j. jclinepi.2017.07.011

13. Stirratt MJ, Dunbar-Jacob J, Crane HM, et al. Self-report measures of medication adherence behavior: recommendations on optimal use. Transl Behav Med. 2015;5(4):470-482. doi:10.1007/s13142-015-0315-2

14. Voils CI, Maciejewski ML, Hoyle RH, et al. Initial validation of a self-report measure of the extent of and reasons for medication nonadherence. Med Care. 2012;50(12):1013. doi:10.1097/MLR.0b01 3e31826b1049

15. Koschack J, Marx G, Schnakenberg J, Kochen MM, Himmel W. Comparison of two self-rating instruments for medication adherence assessment in hypertension revealed insufficient psychometric properties. J Clin Epidemiol. 2010;63(3):299-306. doi:10.1016/j.jclinepi. 2009.06.011

16. Voils CI, King HA, Neelon B, et al. Characterizing weekly self-reported antihypertensive medication nonadherence across repeated occasions. Patient Prefer Adherence. 2014;8:643. doi:10.2147/PPA.S60715

17. Blalock DV, Zullig LL, Bosworth HB, Taylor SS, Voils CI. Self-reported medication nonadherence predicts cholesterol levels over time. $J$ Psychosom Res. 2019;118:49-55. doi:10.1016/j.jpsychores.2019.01.010

18. Morisky DE, Ang A, Krousel-Wood M, Ward HJ. Predictive validity of a medication adherence measure in an outpatient setting. $J$ Clin Hypertens. 2008;10(5):348-354.

19. Voils CI, King H, Thorpe CT, et al. Content validity and reliability of a self-report measure of medication nonadherence in hepatitis $\mathrm{C}$ treatment. Dig Dis Sci. 2019;1-14.
20. Blalock DV, Bosworth HB, Reeve BB, Voils CI. Co-occurring reasons for medication nonadherence within subgroups of patients with hyperlipidemia. J Behav Med. 2018;42(2):1-9.

21. Sutton S, Kinmonth A-L, Hardeman W, et al. Does electronic monitoring influence adherence to medication? Randomized controlled trial of measurement reactivity. Ann Behav Med. 2014;48(3):293299. doi:10.1007/s12160-014-9595-x

22. Vrijens B, De Geest S, Hughes DA, et al. A new taxonomy for describing and defining adherence to medications. $\mathrm{Br} \mathrm{J}$ Clin Pharmacol. 2012;73(5):691-705. doi:10.1111/j.1365-2125.2012.04 167.x

23. Birk J, Kronish I, Chang B, et al. The impact of cardiac-induced posttraumatic stress disorder symptoms on cardiovascular outcomes: design and rationale of the prospective observational Reactions to Acute Care and Hospitalizations (ReACH) Study. Health Psychol Bull. 2019;3(1):10-20. doi:10.5334/hpb.16

24. Simel DL, Rennie D. Pretest probabilities and likelihood ratios for clinical findings. In: DeAngelis $\mathrm{CD}$, editor. The Rational Clinical Examination: Evidence-Based Clinical Diagnosis. New York (NY): McGraw-Hill Education, LLC; 2009.

25. Hayden SR, Brown MD. Likelihood ratio: a powerful tool for incorporating the results of a diagnostic test into clinical decisionmaking. Ann Emerg Med. 1999;33(5):575-580. doi:10.1016/s0196-0644(99) 70346-x

26. Monnette A, Zhang Y, Shao H, Shi L. Concordance of adherence measurement using self-reported adherence questionnaires and medication monitoring devices: an updated review. PharmacoEconomics. 2018;36(1):17-27. doi:10.1007/s40273-017-0570-9

27. Voils CI, Hoyle RH, Thorpe CT, Maciejewski ML, Yancy WS. Improving the measurement of self-reported medication nonadherence. $J$ Clin Epidemiol. 2011;64(3):250-254. doi:10.1016/j.jclinepi.2010.07.014

28. Alfian SD, Pradipta IS, Hak E, Denig P. A systematic review finds inconsistency in the measures used to estimate adherence and persistence to multiple cardiometabolic medications. J Clin Epidemiol. 2019;108:44e53. doi:10.1016/j.jclinepi.2018.12.003
Patient Preference and Adherence

\section{Publish your work in this journal}

Patient Preference and Adherence is an international, peer-reviewed, open access journal that focusing on the growing importance of patient preference and adherence throughout the therapeutic continuum. Patient satisfaction, acceptability, quality of life, compliance, persistence and their role in developing new therapeutic modalities and compounds to optimize clinical outcomes for existing disease states are major areas of interest for the journal. This journal has been accepted for indexing on PubMed Central. The manuscript management system is completely online and includes a very quick and fair peer-review system, which is all easy to use. Visit http:// www.dovepress.com/testimonials.php to read real quotes from published authors. 